Keywords: CD10; head and neck squamous cell carcinoma; cell surface antigen array; chemo-resistance; radio-resistance; cancer stem cells

\title{
CD10 as a novel marker of therapeutic resistance and cancer stem cells in head and neck squamous cell carcinoma
}

\author{
T Fukusumi ${ }^{1}{ }^{1}$, H Ishii ${ }^{2}$, M Konno ${ }^{2}$, T Yasui ${ }^{1}$, S Nakahara ${ }^{1}$, Y Takenaka ${ }^{1}$, Y Yamamoto ${ }^{1}$, S Nishikawa ${ }^{2}$, Y Kano $^{2}$, \\ $\mathrm{H} \mathrm{Ogawa}^{3}$, S Hasegawa ${ }^{3}, \mathrm{~A} \mathrm{Hamabe}^{3}$, N Haraguchi ${ }^{4}, \mathrm{Y} \mathrm{Doki}^{3}, \mathrm{M} \mathrm{Mori}^{3}$ and H Inohara*,1 \\ ${ }^{1}$ Department of Otorhinolaryngology—Head and Neck Surgery, Osaka University, Graduate School of Medicine, Suita, 2-2 \\ Yamadaoka, Osaka 565-0871, Japan; ${ }^{2}$ Department of Frontier Science for Cancer and Chemotherapy, Osaka University, Graduate \\ School of Medicine, Suita, 2-2 Yamadaoka, Osaka 565-0871, Japan; ${ }^{3}$ Department of Gastroenterological Surgery, Osaka University, \\ Graduate School of Medicine, Suita, 2-2 Yamadaoka, Osaka 565-0871, Japan and ${ }^{4}$ Department of Surgery, Osaka National \\ Hospital, Osaka, Tyuou-ku, 2-1-14 Houenzaka, Osaka 540-0006, Japan
}

Background: Cancer stem cells (CSCs) are responsible for treatment failure. However, their identification and roles in resistance are not well established in head and neck squamous cell carcinoma (HNSCC).

Methods: Three HNSCC cell lines (FaDu, Detroit562 and BICR6) were treated with cisplatin or radiation. Cell surface antigens were analysed by LyoPlate, a novel cell surface antigen array. The expression levels of antigens highly expressed after treatments were further compared between cisplatin-resistant Detroit562 cells and its parental line. Association of the candidate antigen with CSCs properties, namely sphere formation and in vivo tumourigenicity, was also examined.

Results: CD10, CD15s, CD146 and CD282 were upregulated across the treated cell lines, while the increased expression of CD10 was prominent in the cisplatin-resistant cell line. Isolation mediated by FACS revealed that the CD10-positive subpopulation was more refractory to cisplatin, fluorouracil and radiation than the CD10-negative subpopulation. It also showed an increased ability to form spheres in vitro and tumours in vivo. Moreover, the CD10-positive subpopulation expressed the CSC marker OCT3/4 at a higher level than that in the CD10-negative subpopulation.

Conclusions: CD10 is associated with therapeutic resistance and CSC-like properties of HNSCC. CD10 may serve as a target molecule in the treatment of refractory HNSCC.

Head and neck squamous cell carcinoma (HNSCC) is the sixth most common malignancy worldwide (Argiris et al, 2008). Despite recent advances in its diagnosis and management, long-term survival of patients with HNSCC remains poor (Lo et al, 2003). Radiotherapy and chemotherapy initially control tumour growth; however, over time many patients suffer relapse. To improve prognosis, the establishment of a novel marker to predict therapeutic resistance is required. This would also aid the optimisation of HNSCC treatment, and thus benefit patient outcome.
Cancer stem cells (CSCs) are defined as cells that possess the properties of tumour initiation and self-renewal. It is currently understood that CSCs are responsible for treatment failure in a diversity of cancers (Bao et al, 2006; Li et al, 2008). CD44 (Prince et al, 2007) and ALDH1 (Chen et al, 2009) have been reported to represent candidate markers of HNSCC CSCs; however, whether they serve as true markers remains controversial (Chen et al, 2011; Koukourakis et al, 2012). These discrepant reports prompted us to search for a novel marker specific to HNSCC CSCs. Thus, in the present study we aimed to identify a new cell surface antigen that is 
involved in therapeutic resistance, and to address whether it served as a marker for HNSCC CSCs. Through array analysis and testing of cell viability in the presence of therapeutic agents, we identified CD10 as a potential marker of refractory HNSCC. Moreover, CD10 was found to confer a CSC-like phenotype, and underscored expression of OCT3/4. Thus, CD10 could be a specific marker of HNSCC CSCs that contributes to therapeutic resistance.

\section{MATERIALS AND METHODS}

Cell culture. FaDu and Detroit562 cell lines were obtained from the ATCC (Manassas, VA, USA), while BICR6 was from ECACC (Proton Down, Salisbury, UK). FaDu and BICR6 were established from a primary hypopharyngeal cancer, while Detroit562 were from a lymph node metastasis of pharyngeal cancer. Cells were cultured in Dulbecco's modified Eagle's medium (DMEM; Sigma Aldrich, St. Louis, MO, USA) supplemented with $10 \%$ foetal bovine serum (FBS) and a penicillin $\left(50 \mathrm{U} \mathrm{ml}^{-1}\right)$ and streptomycin $\left(50 \mu \mathrm{g} \mathrm{ml}^{-1}\right)$ cocktail under an atmosphere of $5 \% \mathrm{CO}_{2}$ at $37^{\circ} \mathrm{C}$. The cisplatin-resistant Detroit562 cell line was established by continuous stepwise exposure to cisplatin starting from a concentration of $1 \mu \mathrm{M}$ up to $10 \mu \mathrm{M}$.

Cell surface antigen arrays. Cells were exposed to $3 \mu \mathrm{m}$ cisplatin for 7 days. Alternatively, cells were irradiated by a single fraction of $8 \mathrm{~Gy}$ and further cultured for 5 days. The expression patterns of cell surface antigens were then compared between the treated and untreated cells using the LyoPlate cell surface antigen array (BD Biosciences, San Jose, CA, USA). The kit consists of three 96-well plates coated with monoclonal antibodies along with AlexaFluor 647 conjugated goat anti-mouse Ig and goat anti-rat Ig secondary antibodies. It allows comprehensive analysis of 242 cell surface antigens by flow cytometry, which was performed using the Cell Analyzer EC800 (Sony, Tokyo, Japan).

Flow cytometry and cell sorting. Flow cytometry and cell sorting were performed using the FACSAria II (BD, Franklin Lakes, NJ, USA). Cells were harvested and single-cell suspensions were prepared with the aid of StemPro Accutase (Life Technologies, Carlsbad, CA, USA). Spheroid cells were separated into single-cell suspensions with the aid of collagenase I (Sigma Aldrich) and adjusted to a concentration of $10^{7}$ cells $\mathrm{ml}^{-1}$. To stain surface antigens, cells were incubated with antibodies against CD10, CD15s, CD44, CD146 and CD282 for $30 \mathrm{~min}$ on ice. The fluorophores for each antibody were as follows: CD10-Brilliant Violet (Biolegend, San Diego, CA, USA) and APC (BD Biosciences); CD44-FITC (BD Biosciences); CD146-APC (Biolegend); CD282-PE (BD Biosciences). For CD15s, we combined purified antibody (BD Biosciences) and the secondary antibodyAPC/Cy7 (BD Biosciences). The antibodies against CD10, CD15s and the secondary antibody of CD15s were used at a concentration of $50 \mu \mathrm{lml}{ }^{-1}$. The antibodies against CD44, CD146 and CD282 were used at a concentration of $200 \mu \mathrm{ml}^{-1}$. To stain ALDH1, we used the Aldefluor stem cell detection kit (StemCell Technologies, Vancouver, BC, Canada) at a concentration of $50 \mu \mathrm{ml}^{-1}$ for $45 \mathrm{~min}$ at $37^{\circ} \mathrm{C}$. The fluorophore of Aldefluor was FITC. Doublet cells were eliminated using FSC-A/FSC-H and SSC-A/SSC-H. Dead and damaged cells were eliminated using 7-AAD (BD Biosciences). Briefly, after CD10, CD15s, CD44, CD146, CD282 and ALDH1 staining, 7-AAD was incubated with cells for $10 \mathrm{~min}$ at room temperature. Except cell sorting, all FACS analysis was performed three times.

Viability assay. Cells were seeded in 96-well plates at $3 \times 10^{3}$ cells per well, cultured overnight and then incubated with $0.1-5 \mu \mathrm{M}$ cisplatin or $0.5-50 \mu \mathrm{M}$ fluorouracil for $72 \mathrm{~h}$. Alternatively, cells were irradiated at a single fraction of $8 \mathrm{~Gy}$ and then cultured for $72 \mathrm{~h}$.
Cell viability was subsequently measured using the Cell Counting Kit-5 (Dojindo Laboratories, Kamimasiki, Japan). The assay was performed three times.

Sphere formation assay. Cells were seeded in 96-well flat bottom ultra-low attachment culture dishes (Corning, Tewksbury, MA, USA) at 10 cells per well in ReproStem medium (ReproCELL, Yokohama, Japan) containing penicillin $\left(50 \mathrm{U} \mathrm{ml}^{-1}\right)$ and streptomycin $\left(50 \mu \mathrm{g} \mathrm{ml}^{-1}\right)$ cocktail and basic fibroblast growth factor $\left(5 \mathrm{ng} \mathrm{ml}^{-1}\right)$ without FBS. After 10 days, the size of spheroid colonies was measured under a microscope and the number of colonies with a diameter over $100 \mu \mathrm{m}$ was counted. The assay was performed three times.

Xenograft assay. The various numbers of cells $\left(1 \times 10^{2}, 1 \times 10^{3}\right.$ and $1 \times 10^{4}$ ) were diluted in equal amounts of DMEM and Matrigel (BD Biosciences) to a final volume of $200 \mu$ l then injected subcutaneously into NOD/SCID mice (Charles River Laboratories Japan, Yokohama, Japan) using a 22-gauge needle. The mice were maintained under pathogen-free conditions and sacrificed 2 months later or when tumours exceeded $20 \mathrm{~mm}$ at the largest diameter. Mice were handled in accordance with the procedures outlined in the Regulations on Animal Experiments at Osaka University. The institutional committee on animal research approved the study.

Quantitative real-time PCR. Quantitative real-time PCR was used to validate siRNA-mediated knockdown of CD10 and to examine mRNA levels of OCT3/4. Briefly, total RNA was isolated from cells using TRIzol reagent (Life Technologies) and cDNA was synthesised using the ReverTra Ace qPCR RT Master Mix (Toyobo, Osaka, Japan). Quantitative reverse transcription-PCR (qRT-PCR) was performed using a Light Cycler TaqMan Master (Roche, Basel, Switzerland). The primer sequences were as follows: CD10 5'-GGGGAGGCTTTATGTGGAAG-3' (sense) and 3'-CTC GGATCTGTGCAATCAAA-5' (antisense); and OCT3/4 5'-GAAA CCCACACTGCAGATCA-3' (sense) and $3^{\prime}$-CGGTTACAGAACC ACACTCG-5' (antisense). Gene expression levels were normalised to that of ACTB, 5'-AGAGCTACGAGCTGCCTGAC-3' (sense) and $3^{\prime}$-CGTGGATGCCACAGGACT-5' (antisense).

Transfection. The siRNA duplexes, si-CD10 and si-control, were obtained from Life Technologies. The si-CD10 sequences were as follows: $5^{\prime}$-GGCCCUUUAUGGUACAACCUCAGAA-3' (sense) and $3^{\prime}$-UUCUGAGGUUGUACCAUAAAGGGCC-5' (antisense). An initial dose-response experiment was performed according to the manufacturer's instructions to determine optimal transfection efficiency. Optimal inhibition was observed at a concentration of $10 \mathrm{~nm}$ siRNA at $72 \mathrm{~h}$ after transfection, thus further qRT-PCR analysis was done under these conditions.

Statistical analysis. The comparison of spheroid colony sizes was made using the Mann-Whitney U-test. The analyses of viability curves were made using two-way analysis of variance. Other statistical comparisons were made using the Student's $t$-test. Differences were considered significant when $P<0.05$. All statistical analyses were performed using JMP Pro 11 (SAS Institute, Cary, NC, USA).

\section{RESULTS}

Identification of antigens related to therapeutic resistance. To identify antigens related to therapeutic resistance, surface antigen expression levels in cells from three HNSCC cell lines, Detroit562, $\mathrm{FaDu}$ and BICR6 that survived treatment with cisplatin or radiation were compared with those of their untreated counterparts by means of LyoPlate (Supplementary Table 1). From this analysis, four cell surface antigens, CD10, CD15s, CD146 and 
CD282, were found to be upregulated in each cell line following either treatment (Table 1).

To further test whether any of these antigens correlated with therapeutic resistance, we established the cisplatin-resistant Detroit562 cell line, which showed excellent viability even in the presence of cisplatin at a concentration as high as $100 \mu \mathrm{M}$ (Figure 1A). The expression levels of CD10, CD15s, CD146 and CD282 were then compared between the parental and cisplatinresistant Detroit562. Only CD10 expression levels were found to be significantly upregulated in cisplatin-resistant Detroit562 when compared with those in the parental line. Indeed, the CD10 $(+)$ subpopulation accounted for $22.5 \%$ compared with $1.4 \%$ in cisplatin-resistant Detroit562 and parental cells, respectively (Figure 1B). Of note, interdependence was not detected among the four markers (Figure 1C). These results indicate that CD10 may serve as a cell surface antigen specific to refractory HNSCC cells.

Association of $\mathrm{CD} 10$ with chemo and radio resistance. To further address the role of CD10 in resistance, we examined whether the $\operatorname{CD} 10(+)$ subpopulation was chemo and/or radio resistant. To do this, $\mathrm{CD} 10(+)$ and $\mathrm{CD} 10(-)$ subpopulations were isolated by FACS from the FaDu and Detroit562 cell lines, and their viability after cisplatin treatment was compared. As shown in Figure $2 \mathrm{~A}$ and $\mathrm{B}$, the $\mathrm{CD} 10(+)$ subpopulation was significantly more refractory to cisplatin than the $\operatorname{CD} 10(-)$ subpopulation in both $\mathrm{FaDu}$ and Detroit562. We also examined whether CD10 affected the sensitivity of cells to fluorouracil, which is used in combination with cisplatin in the treatment of HNSCC (Kish et al, 1982). As shown in Figure 2C and D, the CD10 $(+)$ subpopulation was also significantly more refractory to fluorouracil in Detroit562. Next, we investigated the association between CD10 and radiation sensitivity. We found that the $\mathrm{CD} 10(+)$ subpopulations of both $\mathrm{FaDu}$ and Detroit562 were significantly more radio resistant than the respective $\mathrm{CD} 10(-)$ subpopulations (Figure 2E).

Association between CD10 and the cell cycle. Generally, cisplatin and fluorouracil affect DNA synthesis. Thus, slow-cell cycling or dormant cells (G0/G1 phase) are resistant to these chemotherapeutic agents (Barr et al, 2013). As for radiation, cells are most sensitive to its effects during the G2/M phase and less sensitive in G1/0 and S phases (Sinclair, 1968). We hypothesised that the chemo and radio resistance of the $\mathrm{CD} 10(+)$ subpopulation was associated with cell cycle phase. Thus, we performed cell cycle analysis using Hoechst33342. As shown Figure 2F-G, the CD10 $(+)$ subpopulation had a greater proportion of cells in the G0/G1 phase and less in the G2/M phase than the CD10(-) subpopulation. These data indicated that the $\mathrm{CD} 10(+)$ subpopulation of HNSCC cells was slow-cell cycling or dormant compared with the CD10(-) subpopulation.
CD10 and sphere formation ability. Given that CSCs are responsible for therapeutic resistance (Bao et al, 2006; Li et al, 2008), and are also in the dormant or slow-growing phase of the cell cycle (Holyoake et al, 1999), we hypothesised that CD10 might be a novel marker for CSCs in HNSCC. One of the most important characteristics of CSCs is self-renewal ability, which is assessed by sphere formation. First, we examined the distribution of CD10 in spheroid cells and control adherent cells using FACS analysis. In $\mathrm{FaDu}, 10.3 \%$ of spheroid cells and $2.1 \%$ of adherent cells were CD10 $(+)$. Similarly, $10.2 \%$ of spheroid cells and $1.7 \%$ of adherent cells were $\mathrm{CD} 10(+)$ in Detroit562 (Figure $3 \mathrm{~A}$ and B). Next, we compared sphere formation ability between $\mathrm{CD} 10(+)$ and $\mathrm{CD} 10(-)$ subpopulations. Although the morphology of spheroid colonies was similar between the two subpopulations (Figure 3C), there was a significant difference in their number. The CD10 $(+)$ subpopulation formed more spheroid colonies than the $\mathrm{CD} 10(-)$ subpopulation in both $\mathrm{FaDu}$ and Detroit562 (Figure 3D-E). Moreover, colonies of the $\mathrm{CD} 10(+)$ subpopulation were larger than those of the CD10 (-) subpopulation in FaDu and Detroit562 (Figure 3F-G).

CD10 and tumourigenicity. To further address the association between CD10 and CSC properties, we examined whether CD10 modulates in vivo tumourigenicity. $\mathrm{CD} 10(+)$ and $\mathrm{CD} 10(-)$ subpopulations were sorted and individually transplanted into NOD/SCID mice. The result of the limiting dilution transplantation assay of Detroit562 cells is shown in Table 2. Briefly, when 1000 cells were transplanted, the $\operatorname{CD} 10(+)$ subpopulation formed tumours in six of six (100\%) transplanted mice, while the CD10(-) subpopulation formed tumours in only two of six $(33 \%)$ mice. Moreover, the $\mathrm{CD} 10(+)$ subpopulation remained tumourigenic with as few as 100 cells. In contrast, there was no difference in tumourigenicity between the $\mathrm{CD} 10(+)$ and $\mathrm{CD} 10(-)$ subpopulations of $\mathrm{FaDu}$ (Supplementary Table 2), although the size of tumours formed by inoculation of 1000 cells was notably larger in the $\mathrm{CD} 10(+)$ subpopulation than in the CD10 $(-)$ subpopulation (Supplementary Figure 1). To confirm that the histology of tumours was squamous cell carcinoma, we performed $\mathrm{H} \& \mathrm{E}$ staining (Figure 4A). Both $\mathrm{FaDu}$ and Detroit562 tumours from $\mathrm{CD} 10(+)$ and $\mathrm{CD} 10(-)$ subpopulations presented with squamous cell carcinoma histology and the shapes of these tumour cells were similar to those of parental cell lines.

Interrelations between CD10 and other CSC markers. It has been reported that CD44 (Prince et al, 2007), CD133 (Chiou et al, 2008) and ALDH1 (Chen et al, 2009) are markers of CSCs in HNSCC; thus, we examined the interdependence between CD10 and these markers. Since CD133 is not contained in the cell surface antigen array, we first assessed its expression in treated (cisplatin or radiation) and untreated FaDu cells by flow cytometry as per the conditions used in the array analysis. We found that CD133 expression was barely detectable even after the treatments

Table 1. Differentially expressed cell surface antigens in three HNSCC cell lines, Detroit562, FaDu and BICR6 following treatment with radiation or cisplatin

\begin{tabular}{|l|c|c|c|c|c|c|c|c|c|}
\hline \multicolumn{7}{|c|}{ Detroit562 } \\
\cline { 2 - 9 }
\end{tabular}



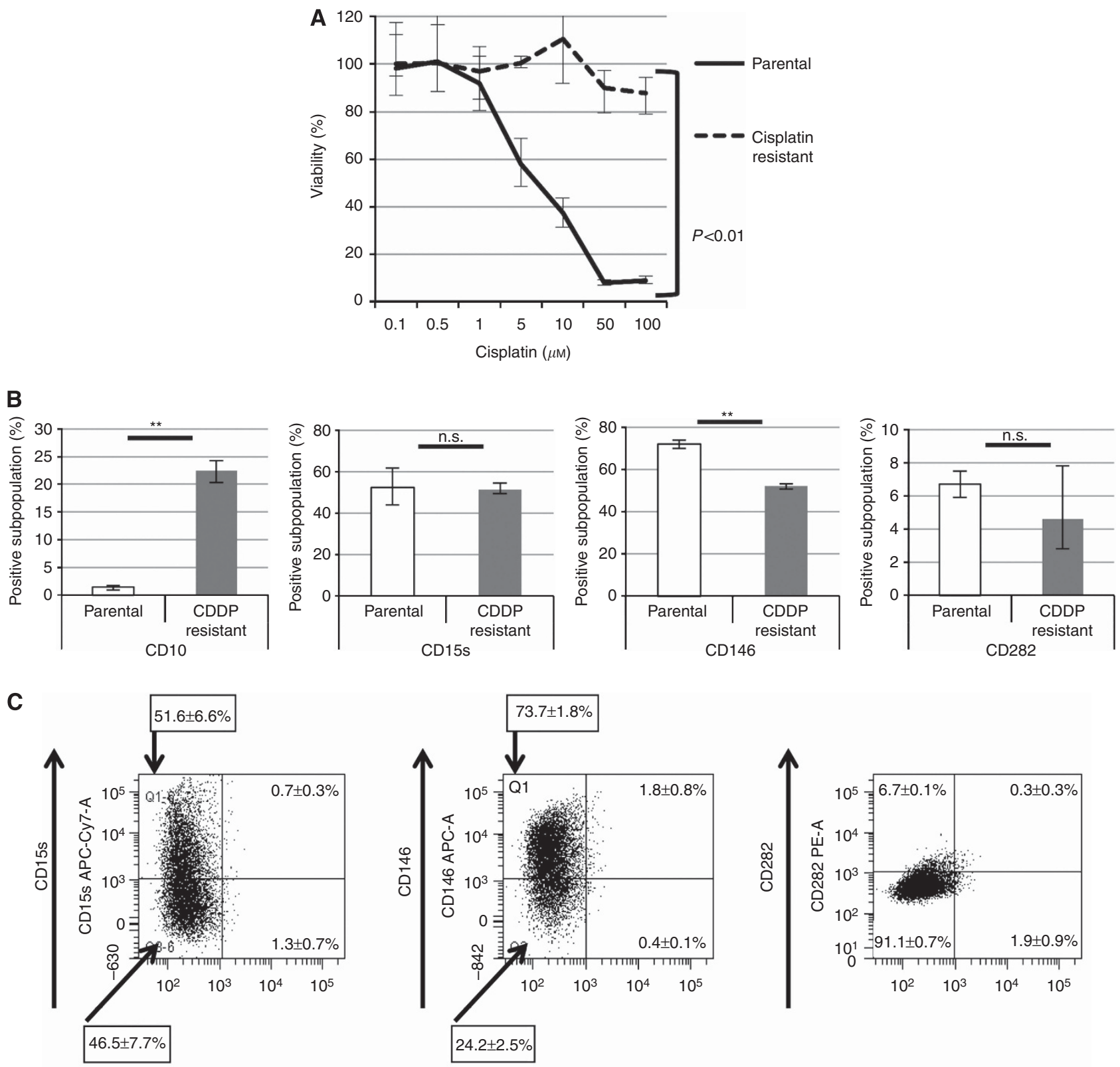

CD10

Figure 1. Expressions of candidate antigens in the cisplatin-resistant HNSCC cell line Detroit562. (A) Cisplatin-resistant Detroit562 were generated and validated by testing their viability against that of the parental cell line in response to cisplatin treatment, at the indicated doses. Statistical analysis was performed using two-way analysis of variance. Data represent means \pm s.e.m. (B) Expression analyses of CD10, CD15s, CD146 and CD282 by flow cytometry in cisplatin-resistant Detroit562 and the parental cells. The percentages of positive subpopulations are indicated; ${ }^{* \star} P<0.01$; n.s., not significant. (C) The interdependence of the four markers.

(Supplementary Table 3). Thus a relationship between CD10 and CD133 by means of FACS analysis could not be explored. As for $\mathrm{CD} 44$, We found that the majority of FaDu and Detroit562 cells were $\operatorname{CD} 44(+)$. Although we found that all $\operatorname{CD} 10(+)$ cells expressed $\mathrm{CD} 44$ in both Detroit562 and $\mathrm{FaDu}$ cell lines (Supplementary Figure 2A), significant interdependence was not detected. As for ALDH1, we found that CD10 $(+)$ cells expressed significantly more ALDH1 than $\mathrm{CD} 10(-)$ counterparts in both cell lines (Figure 4B and Supplementary Figure 2B). The expression levels of CD10 and ALDH1 were found to be interdependent.

Stem cell-related genes in CD10-positive cells. To shed light on the molecular mechanisms underlying self-renewal ability and tumourigenicity of the $\mathrm{CD} 10(+)$ subpopulation, we compared the expression of OCT3/4, a known marker of tissue stem cells (Nichols et al, 1998) and CSCs (Nichols et al, 1998), between $\mathrm{CD} 10(+)$ and $\mathrm{CD} 10(-)$ subpopulations. OCT3/4 expression was significantly increased in the $\operatorname{CD} 10(+)$ subpopulation when compared with that of the $\mathrm{CD} 10(-)$ subpopulation in both $\mathrm{FaDu}$ and Detroit562 (Figure 4C). Of note, knockdown of CD10 by siRNA resulted in decreased expression of OCT3/4 (Figure 4D and Supplementary Figure 3A-B).

\section{DISCUSSION}

In the present study, we used the novel cell surface antigens array Lyoplate to identify antigens relevant to cell survival after 

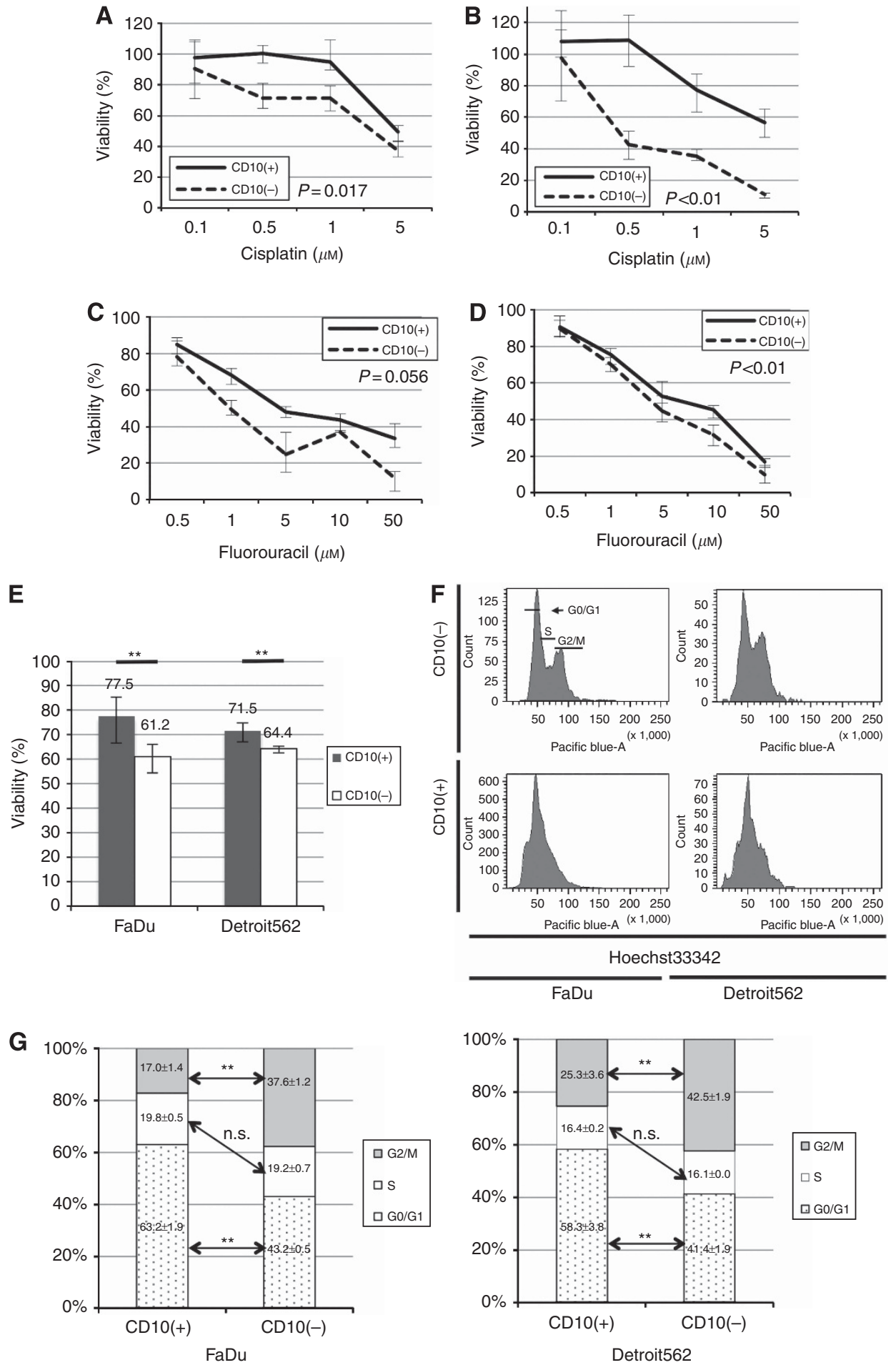

Figure 2. $\mathrm{CD} 10(+) /(-)$ cell viability in response to treatment with cisplatin, fluorouracil, or radiation and cell cycle analysis. (A-D) CD10(+) and CD10( - ) subpopulations were isolated by FACS and cultured with $0.1-5 \mu \mathrm{m}$ cisplatin or $0.5-50 \mu \mathrm{m}$ fluorouracil for $72 \mathrm{~h}$. Viability in response to cisplatin in FaDu (A) and Detroit562 (B) or fluorouracil in FaDu (C) and Detroit562 (D) was then measured. Statistical analysis was performed using two-way analysis of variance. (E) Alternatively, cells were exposed to radiation at single fraction $8 \mathrm{~Gy}$. After $72 \mathrm{~h}$, cell viability was measured. Data represent means \pm s.e.m.; ${ }^{\star \star} P<0.01$. (F) Cell cycle analysis of CD10-positive and -negative subpopulations was performed after staining with Hoechst33342. (G) The cell cycle phase distribution of CD10 $(+) /(-)$ subpopulations. ${ }^{\star \star} P<0.01$; n.s., not significant.

treatment with cisplatin or radiation. This is the first report that tries to identify an antigen that exhibits both therapeutic resistance and is related to CSCs by means of the cell surface antigens array. We found that CD10, CD15s, CD146 and CD282 were highly expressed in treated cells compared with untreated cells. To validate the result of the cell surface antigens array, we next compared the expression of these antigens between a cisplatin-resistant cell line and its parental cell line. Of the candidate antigens, only expression of CD10 was upregulated in the cisplatin-resistant cell line as determined by FACS analysis. We propose two reasons for the different antigen expression profiles detected by Lyoplate and FACS analysis. First, different flow cytometers were used for the detection of signals, thus variations in sensitivity may account for the divergent findings. 

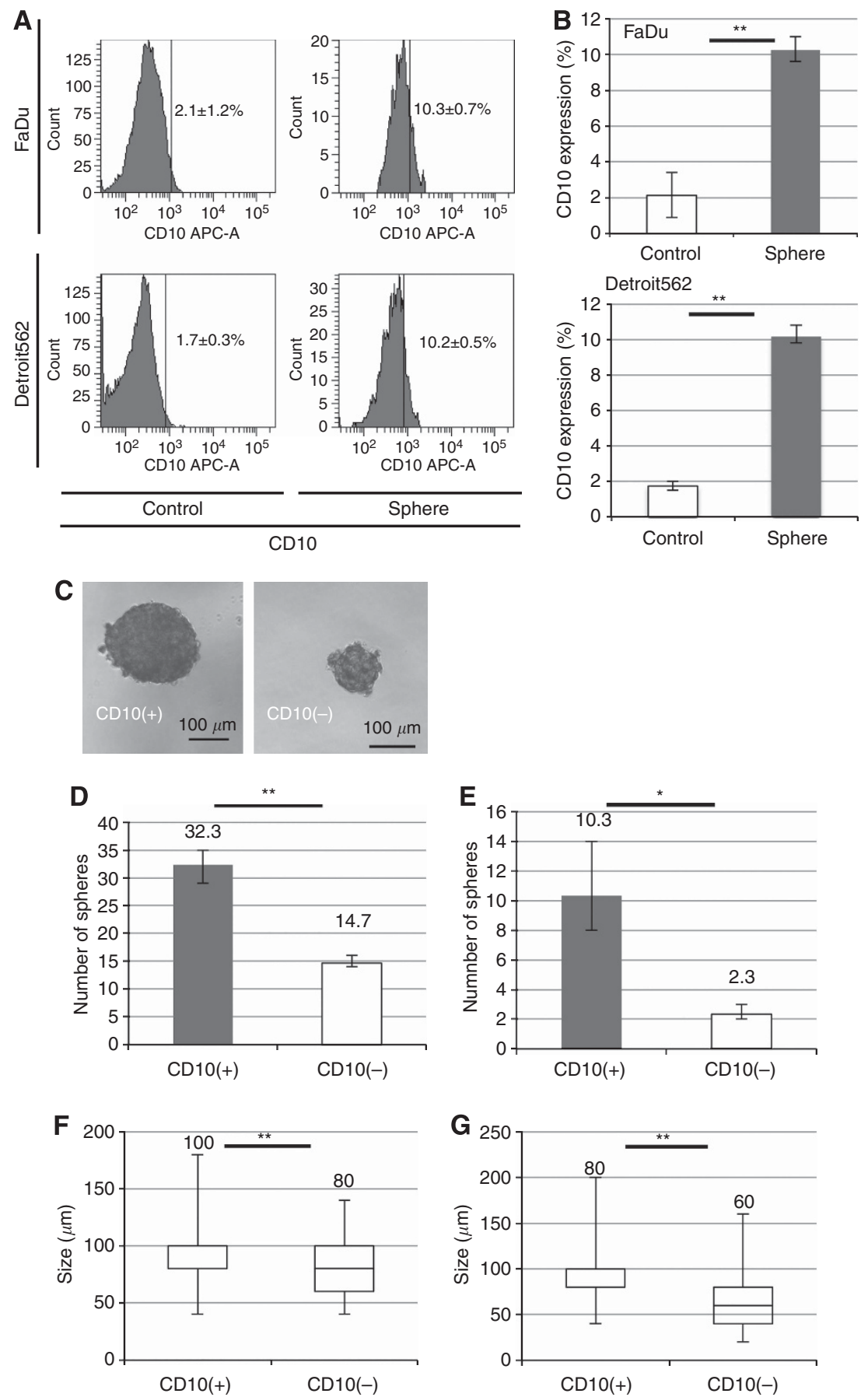

Figure 3. CD10 and sphere formation. (A-B) CD10 expression in sphere cells was compared with that of adherent (control) cells in FaDu and Detroit562 by flow cytometry. (C) CD10(+) and CD10(-) cells were sorted and the morphology of spheroid colonies in FaDu were examined. Representative images are shown. (D-E) The number of spheroid colonies formed in CD10(+)/( - ) FaDu (D) and Detroit562 (E) were calculated. $(\mathbf{F}-\mathbf{G})$ The sizes of spherical colonies of $\mathrm{CD} 10(+) /(-)$ in $\mathrm{FaDu}(\mathbf{F})$ and Detroit562 (G) were also determined. Data represent means \pm s.e.m.; ${ }^{\star} P<0.05 ;{ }^{\star *} P<0.01$.

Table 2. Tumourigenicity of $\mathrm{CD} 10(+)$ and $\mathrm{CD} 10(-)$ Detroit562 cells

\begin{tabular}{|l|c|c|}
\hline $\begin{array}{l}\text { No. of cells used } \\
\text { for inoculation }\end{array}$ & $\begin{array}{c}\text { No. of tumours } \\
\text { from CD10 }(+) \text { cells }\end{array}$ & $\begin{array}{c}\text { No. of tumours } \\
\text { from CD10 }(-) \text { cells }\end{array}$ \\
\hline 10000 & $4 / 4$ & $4 / 4$ \\
\hline 1000 & $6 / 6$ & $2 / 6$ \\
\hline 100 & $1 / 4$ & $0 / 4$ \\
\hline
\end{tabular}

Second, it is the difference of products of antibodies such as clone number, type of fluorophores and method of staining. These may further underlie differences in technical sensitivity. However, both techniques clearly demonstrated that CD10 was upregulated in response to either cisplatin or radiation treatment, as well as in the cisplatin-resistant cell line.

CD10, also known as membrane metalloendopeptidase, neutral endopeptidase, neprilysin and common acute lymphoblastic leukaemia antigen (CALLA), is a zinc-dependent 

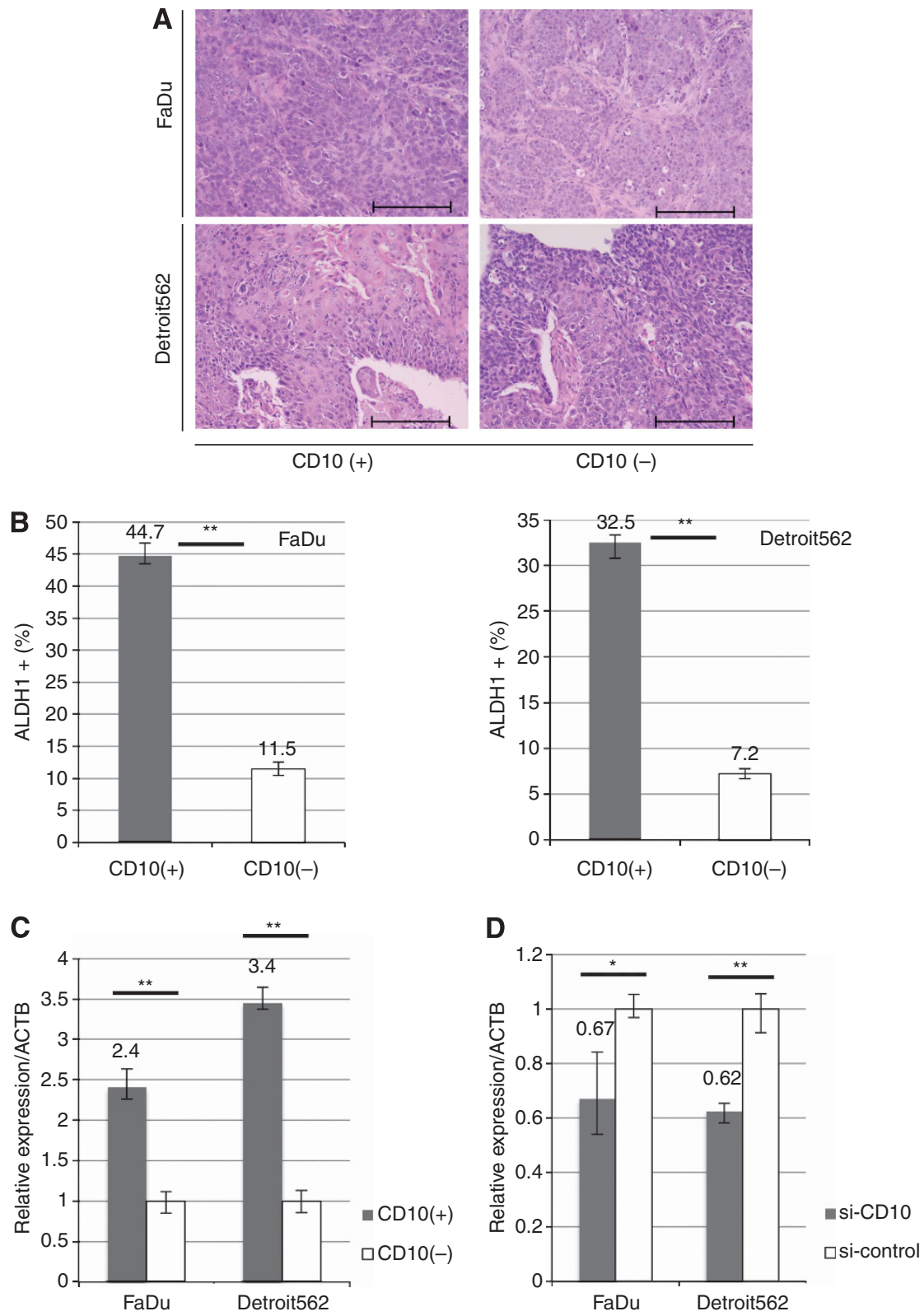

Figure 4. Histology of tumours from $\mathrm{CD} 10(+) /(-)$ subpopulations and the relationship between $\mathrm{CD} 10$ and other stem cell markers. (A) H\&E staining of FaDu and Detroit562 xenograft tumours. Scale bar, $100 \mu \mathrm{M}$. (B) Expression of ALDH1 in CD10 $(+) /(-)$ FaDu and Detroit562 cells was assessed by FACS. Data represent means \pm s.e.m.; ${ }^{\star \star} P<0.01$. (C) OCT3/4 expression in CD $10(+) /(-)$ FaDu and Detroit562 cells was assessed by qRT-PCR. (D) OCT3/4 expression in FaDu and Detroit562 following transfection with either si-CD10 or si-control was assessed by qRT-PCR. Gene expression levels are presented as a ratio of the internal control, ACTB \pm s.e.m. ${ }^{\star} P<0.05 ;{ }^{*} P<0.01$.

metalloendoprotease that cleaves signalling peptides (Roques et al, 1993; Turner \& Tanzawa, 1997). It is expressed in a wide range of normal cells, and has been shown to be a cell surface marker of tissue stem cells in the bone marrow (Galy et al, 1998), adipose (Buhring et al, 2007), lung (Sunday et al, 1992) and breast (Stingl et al, 2005). CD10 is also expressed in a series of malignancies originating from the kidney, lung, skin, pancreas, prostate, liver, breast, stomach, cervix and bladder. Several studies have shown an association between CD10 and metastasis (Maguer-Satta et al, 2011). In HNSCC, an involvement of CD10 in tumour differentiation and growth has been reported (Piattelli et al, 2006). This report also showed that expression of CD10 was associated with distant metastases, local recurrences and histological grade in HNSCC patients. Because of this background and our experiments, we hypothesised that CD10 is a marker for refractory HNSCC. Thus, we further examined whether the CD10-positive subpopulation was chemo and/or radio resistant. We found that the CD10-positive subpopulation was more resistant to treatment with cisplatin, fluorouracil or radiation in comparison with the CD10-negative subpopulation.

Several mechanisms, such as efficient DNA repair and expression of transporter pumps, as well as changes in cell cycling are considered to explain such resistance. Among these mechanisms, we focussed on the cell cycle. We analysed cell cycle phase distributions between CD10-positive and CD10-negative subpopulations. We found that the percentage of G0/G1 phase cells was increased in CD10-positive subpopulation when compared with that of the CD10-negative subpopulation. This result indicates that the CD10-positive subpopulation of HNSCC cells was slow-cell cycling or dormant compared with CD10-negative subpopulation.

Recent studies have shown that CSCs are responsible for the therapeutic resistance of cancers (Bao et al, 2006; Li et al, 2008). 
Additionally, CSCs are slow-cycling or in the dormant phase of the cell cycle. For example, CSCs of acute myeloid leukaemia (Guan et al, 2003) and chronic myeloid leukaemia (Holyoake et al, 1999) survive in the dormant G0 phase of the cell cycle. In the case of solid tumours, liver CSCs were found to be mainly in the G0/G1 phase (Haraguchi et al, 2010). Thus, we addressed the relevance of CD10 for the CSC phenotype. We found that the CD10-positive subpopulation formed spheres in vitro and tumours in vivo more efficiently than the CD10-negative subpopulation. These results indicate that CD10 is closely related to tumourigenicity and selfrenewal ability. Thus, it seems likely that CD10 could serve as a marker of CSCs in HNSCC.

Previously, CD44 (Prince et al, 2007), CD133 (Chiou et al, 2008) and ALDH1 (Chen et al, 2009) have been reported as markers of CSCs in HNSCC. However, whether CD44 and ALDH1 serve as true markers remains controversial. For instance, recent studies have shown that decreased rather than increased expression of ALDH1 is linked to poor prognosis (Koukourakis et al, 2012), while CD44 is expressed in normal head and neck squamous epithelium at an equivalent level to that detected in HNSCC (Chen et al, 2011). As for therapeutic resistance, CD44 expression was downregulated in irradiated cells when compared with that of untreated cells as determined in our cell surface antigen array assay of the HNSCC cell line BICR6. Moreover, the vast majority of $\mathrm{FaDu}$ and Detroit562 cells were CD44-positive irrespective of cisplatin or radiation treatment. In the case of CD133, Zhang et al, (2010) showed that CD133-positive cells possessed resistance to paclitaxel when compared with CD133-negative cells in oral cancer cell lines, although CD133 expression was barely detectable, even after the treatment, in the cell lines used in our study. Together, these data indicate that neither CD44 nor CD133 have pivotal roles in the therapeutic resistance of these HNSCC cell lines. Although it cannot be dismissed that the relevance of CD44 and CD133 in therapeutic resistance probably depends on the kinds of cells lines and treatments administered. Thus, further study is needed to determine the relationship between CSC-related properties and therapeutic resistant in HNSCC, including investigation into the effect of combination of CD10 and these markers (CD44, CD133, ALDH1). Especially, we consider the combination of CD10 and ALDH1, because we found these interdependent expressions.

Notably, we demonstrated that the CD10-positive subpopulation of HNSCC cells showed CSC-related properties, such as chemo and radio resistance, self-renewal capacity and tumourigenicity. To gain insight into the mechanisms by which CD10 confers CSC-related properties in HNSCC, we examined the expression of OCT3/4, which has a critical role in the development and self-renewal of embryonic stem cells (Nichols et al, 1998), and is linked to oncogenic processes (Gidekel et al, 2003). Chen et al, (2010, 2011). have shown that OCT3/4 is upregulated in HNSCC CSCs, defined by ALDH1 positive cells, and in spheroid forming HNSCC cells. We found that OCT3/4 expression was higher in CD10-positive cells than in CD10-negative cells, but that it was decreased following knockdown of CD10. These results indicate that increased CD10 is linked to OCT3/4 expression. Further studies are required to address the functional relevance of CD10 to OCT3/4 in HNSCC.

In conclusion, we have established that CD10 is associated with chemo and radio resistance, and that it confers CSC-related properties in HNSCC, probably through forced overexpression of OCT3/4. Together these findings suggest that CD10 may serve as a target molecule in the treatment of refractory HNSCC.

\section{ACKNOWLEDGEMENTS}

We thank Miyuki Ozaki and Yuko Noguchi for their technical support. The current study was partly funded by a Core Research
Grant-in-Aid for Scientific Research from the Ministry of Education, Culture, Sports, Science and Technology, Japan; a Grant-in-Aid from the Third Term Comprehensive 10-year Strategy for Cancer Control of the Ministry of Health, Labour and Welfare, Japan; and grants from the Kobayashi Cancer Research Foundation, the Princess Takamatsu Cancer Research Fund, Japan; the SENSHIN Medical Research Foundation, Japan; and the National Institute of Biomedical Innovation, Japan.

\section{REFERENCES}

Argiris A, Karamouzis MV, Raben D, Ferris RL (2008) Head and neck cancer. Lancet 371(9625): 1695-1709.

Bao S, Wu Q, McLendon RE, Hao Y, Shi Q, Hjelmeland AB, Dewhirst MW, Bigner DD, Rich JN (2006) Glioma stem cells promote radioresistance by preferential activation of the DNA damage response. Nature 444(7120): 756-760.

Barr MP, Gray SG, Hoffmann AC, Hilger RA, Thomale J, O’Flaherty JD, Fennell DA, Richard D, O'Leary JJ, O’Byrne KJ (2013) Generation and characterisation of cisplatin-resistant non-small cell lung cancer cell lines displaying a stem-like signature. PloS one 8(1): e54193.

Buhring HJ, Battula VL, Treml S, Schewe B, Kanz L, Vogel W (2007) Novel markers for the prospective isolation of human MSC. Ann NY Acad Sci 1106: 262-271.

Chen C, Wei Y, Hummel M, Hoffmann TK, Gross M, Kaufmann AM, Albers AE (2011) Evidence for epithelial-mesenchymal transition in cancer stem cells of head and neck squamous cell carcinoma. PloS one 6(1): e16466.

Chen YC, Chang CJ, Hsu HS, Chen YW, Tai LK, Tseng LM, Chiou GY, Chang SC, Kao SY, Chiou SH, Lo WL (2010) Inhibition of tumorigenicity and enhancement of radiochemosensitivity in head and neck squamous cell cancer-derived ALDH1-positive cells by knockdown of Bmi-1. Oral Oncol 46(3): 158-165.

Chen YC, Chen YW, Hsu HS, Tseng LM, Huang PI, Lu KH, Chen DT, Tai LK, Yung MC, Chang SC, Ku HH, Chiou SH, Lo WL (2009) Aldehyde dehydrogenase 1 is a putative marker for cancer stem cells in head and neck squamous cancer. Biochem Biophys Res Commun 385(3): 307-313.

Chiou SH, Yu CC, Huang CY, Lin SC, Liu CJ, Tsai TH, Chou SH, Chien CS, $\mathrm{Ku} \mathrm{HH}$, Lo JF (2008) Positive correlations of Oct-4 and Nanog in oral cancer stem-like cells and high-grade oral squamous cell carcinoma. Clin Cancer Res 14(13): 4085-4095.

Galy A, Morel F, Hill B, Chen BP (1998) Hematopoietic progenitor cells of lymphocytes and dendritic cells. J Immunother 21(2): 132-141.

Gidekel S, Pizov G, Bergman Y, Pikarsky E (2003) Oct-3/4 is a dosedependent oncogenic fate determinant. Cancer Cell 4(5): 361-370.

Guan Y, Gerhard B, Hogge DE (2003) Detection, isolation, and stimulation of quiescent primitive leukemic progenitor cells from patients with acute myeloid leukemia (AML). Blood 101(8): 3142-3149.

Haraguchi N, Ishii H, Mimori K, Tanaka F, Ohkuma M, Kim HM, Akita H, Takiuchi D, Hatano H, Nagano H, Barnard GF, Doki Y, Mori M (2010) CD13 is a therapeutic target in human liver cancer stem cells. J Clin Invest 120(9): 3326-3339.

Holyoake T, Jiang X, Eaves C, Eaves A (1999) Isolation of a highly quiescent subpopulation of primitive leukemic cells in chronic myeloid leukemia. Blood 94(6): 2056-2064.

Kish J, Drelichman A, Jacobs J, Hoschner J, Kinzie J, Loh J, Weaver A, Al-Sarraf M (1982) Clinical trial of cisplatin and 5-FU infusion as initial treatment for advanced squamous cell carcinoma of the head and neck. Cancer Treat Rep 66(3): 471-474.

Koukourakis MI, Giatromanolaki A, Tsakmaki V, Danielidis V, Sivridis E (2012) Cancer stem cell phenotype relates to radio-chemotherapy outcome in locally advanced squamous cell head-neck cancer. Br J Cancer 106(5): 846-853.

Li X, Lewis MT, Huang J, Gutierrez C, Osborne CK, Wu MF, Hilsenbeck SG, Pavlick A, Zhang X, Chamness GC, Wong H, Rosen J, Chang JC (2008) Intrinsic resistance of tumorigenic breast cancer cells to chemotherapy. J Natl Cancer Inst 100(9): 672-679.

Lo WL, Kao SY, Chi LY, Wong YK, Chang RC (2003) Outcomes of oral squamous cell carcinoma in Taiwan after surgical therapy: factors affecting survival. J Oral Maxillofac Surg 61(7): 751-758. 
Maguer-Satta V, Besancon R, Bachelard-Cascales E (2011) Concise review: neutral endopeptidase (CD10): a multifaceted environment actor in stem cells, physiological mechanisms, and cancer. Stem Cells 29(3): 389-396.

Nichols J, Zevnik B, Anastassiadis K, Niwa H, Klewe-Nebenius D, Chambers I, Scholer H, Smith A (1998) Formation of pluripotent stem cells in the mammalian embryo depends on the POU transcription factor Oct4. Cell 95(3): 379-391.

Piattelli A, Fioroni M, Iezzi G, Perrotti V, Stellini E, Piattelli M, Rubini C (2006) CD10 expression in stromal cells of oral cavity squamous cell carcinoma: a clinic and pathologic correlation. Oral Dis 12(3): 301-304.

Prince ME, Sivanandan R, Kaczorowski A, Wolf GT, Kaplan MJ, Dalerba P, Weissman IL, Clarke MF, Ailles LE (2007) Identification of a subpopulation of cells with cancer stem cell properties in head and neck squamous cell carcinoma. Proc Natl Acad Sci USA 104(3): 973-978.

Roques BP, Noble F, Dauge V, Fournie-Zaluski MC, Beaumont A (1993) Neutral endopeptidase 24.11: structure, inhibition, and experimental and clinical pharmacology. Pharmacol Rev 45(1): 87-146.
Sinclair WK (1968) Cyclic X-ray responses in mammalian cells in vitro. Radiation research 33: 620-643.

Stingl J, Raouf A, Emerman JT, Eaves CJ (2005) Epithelial progenitors in the normal human mammary gland. J Mammary Gland Biol Neoplasia 10(1): 49-59.

Sunday ME, Hua J, Torday JS, Reyes B, Shipp MA (1992) CD10/neutral endopeptidase 24.11 in developing human fetal lung. Patterns of expression and modulation of peptide-mediated proliferation. J Clin Invest 90(6): 2517-2525.

Turner AJ, Tanzawa K (1997) Mammalian membrane metallopeptidases: NEP, ECE, KELL, and PEX. FASEB J 11(5): 355-364.

Zhang Q, Shi S, Yen Y, Brown J, Ta JQ, Le AD (2010) A subpopulation of CD133( + ) cancer stem-like cells characterized in human oral squamous cell carcinoma confer resistance to chemotherapy. Cancer Lett 289(2): 151-160.

This work is published under the standard license to publish agreement. After 12 months the work will become freely available and the license terms will switch to a Creative Commons AttributionNonCommercial-Share Alike 3.0 Unported License.

Supplementary Information accompanies this paper on British Journal of Cancer website (http://www.nature.com/bjc) 